\title{
THE EFFECT OF THE SPARK ON THE OSCILLATIONS OF AN ELECTRIC CIRCUIT*
}

\author{
$\mathrm{By}$ \\ John Stone Stone \\ (Congulting Electrical and Radio Engineer, Past President of The \\ Institute of Radio EngineERs)
}

When a condenser is permitted to discharge thru a coil under such conditions as not to produce a spark or under such conditions that the resistance of the spark is completely negligible compared to the conductor resistance of the circuit, the phenomena observed accord well with the mathematical theory advanced in 1853 by Professor William Thompson, the late Lord Kelvin. ${ }^{1}$ When, on the other hand, the discharge of the condenser is accompanied by the spark and the conditions are such that the conductor resistance of the circuit is negligible compared to the resistance of the spark, then the Thompson theory no longer applies, even approximately, and such oscillations as occur in the circuit are of a character, the mathematical theory of which, I had the honor to present to this Institute in $1914 .^{2}$

The Thompson theory, which we may best describe as the logarithmic decrement theory, is therefore seen to be the mathematical theory of one extreme or limiting case of electrical oscillation. This extreme case I have shown to be characteristic of very low frequency oscillators. The theory which I presented to you last Spring and which may best be described as the linear decrement theory, is seen to be the mathematical theory of an equally extreme case of electrical oscillation-a case which I have shown to appertain particularly to very high frequency oscillators.

Tho most oscillating circuits used in radio telegraphy are of very high frequency, nevertheless, there are many in which the conductor resistance is by no means negligible compared to the resistance of the spark and such electric oscillators therefore fall into the class for which there is as yet no competent theory upon

* Presidential Address presented before The Institute of Radio Engineers, New York, February 3, 1915.

1"Phil. Mag.," Series 4, Volume V, page 393.

2 Proceedings of The Institute of Radio Engineers, Volume 2, Number 4, page 307. 
which reliable quantitative measurements may be based or from which reliable deductions may be made. It has seemed to me that it might therefore be desirable to carry the investigation of the effect of the spark upon the oscillations in an electric circuit further than was done in my latest paper and to consider the more general theory in which no limitation is placed upon the relative magnitude of the conductor and spark resistances. Such a general theory, of course, includes the logarithmic decrement theory and the linear decrement theory as special and oppositely extreme limiting cases.

It might well be expected that the deduction of this general theory would involve grave difficulties and that the resulting expressions deduced for the oscillatory charge, voltage, and current would be of considerable complexity. Neither of these sources of inconvenience has, however, been found to exist. The great simplicity both of deduction and result flows directly from the fact that the resistance of the spark is in general inversely proportional to the amplitude of the oscillating current flowing thru the spark, provided the oscillations be of radio frequencies and the spark gap electrodes be not composed of the so-called nonarcing materials. This fact was shown, in my before-mentioned paper, to be one of the necessary consequences of the experimentally observed linear mode of decay of the oscillations, noted when the spark resistance is the dominant resistance of the circuit.

As this paper is practically a second installment of my latest paper on this subject before the Institute, it is not necessary to repeat here the numerous collateral statements which it would otherwise be expedient to make by way of explanation, definition and limitation, and we may therefore proceed directly to the solution of the circuital equation:

$$
0=\frac{1}{C} \int i d t+R i+L \frac{d i}{d t}
$$

where $C$ is the capacity of the condenser, $i$ is the current at any moment, $R$ is the total resistance of the circuit at that moment and $L$ is the inductance of the circuit.

Let $R_{1}$ be the constant conductor-resistance of the circuit and $R_{2}$ be the spark resistance.

By dividing (1) thru by $L$, we may write the equation as:

$$
0=\omega_{0}^{2} \int i d t+2\left(\alpha_{1}+\alpha_{2}\right) i+\frac{d i}{d t}
$$

where

$$
\omega_{o}^{2}=\frac{1}{C L}, \alpha_{1}=\frac{R_{1}}{2 L} \text { and } \alpha_{2}=\frac{R_{2}}{2 L}
$$


We know that the current is of the form $i=I \sin (\omega t+\psi)$ where $\omega$ and $\psi$ are constant and $I$ only is a function of $t$ because the most careful resonance analysis of these oscillators fails to reveal the presence of a second frequency. If the spark introduced an oscillation of a second frequency, its presence could be detected even tho its amplitude were less than 1 per cent. of that of the principal or fundamental oscillation except in the case of extremely high damping. Furthermore, since the current must be zero when $t$ is zero, if $t=0$, be chosen as the time at which the oscillations begin, then

$$
i=I \sin \omega t
$$

It follows from what has been said above and from what was shown in my last paper, that

$$
R_{2}=\frac{d}{I}
$$

where $d$ is a constant.

Substituting these values for $i$ and $R_{2}$ in the third term of the right hand number of (2) gives

$$
-\frac{d}{L} \sin \omega t=\omega_{o}^{2} \int i d t+2 \alpha_{1} i+\frac{d i}{d t}
$$

We see immediately that the effect of the spark in the circuit is merely to introduce a simple harmonic counter electro-motive force $-\frac{d}{L} \sin \omega t$ into the circuit at the beginning of the oscillations and to maintain it there at constant amplitude $-\frac{d}{L}$ as long as the oscillations last.

The solution of (3) therefore resolves itself into the solution of two equations:

and

$$
0=\omega_{o}^{2} \int i_{1} d t+2 \alpha_{1} i_{1}+\frac{d i_{1}}{d t}
$$

where $i_{1}+i_{2}=i$.

$$
-\frac{d}{L} \sin \omega t=\omega_{o}^{2} \int i_{2} d t+2 a_{1} i_{2}+\frac{d i_{2}}{d t}
$$

The complete solution of (4) with all constants determined by the conditions, $i_{1}=0$ and $\int i_{1} d t=Q_{o}$ at $t=0$ is:

$$
i_{1}=I_{1} \varepsilon^{-a t} \sin \omega t
$$

where

and

$$
\begin{aligned}
& I_{1}=Q_{o} \frac{\alpha^{2}+\omega^{2}}{\omega} \equiv Q_{o} \frac{\omega_{o}^{2}}{\omega}, \\
& \alpha=\alpha_{1} \\
& \omega^{2}=\omega_{o}^{2}-\alpha^{2} .
\end{aligned}
$$


The solution of ( $\mathbf{5})$ is:

$$
-I_{2} \sin (\omega t+\theta)+I_{3} z^{-a_{1} t} \sin (\omega t+\phi)
$$

where $\quad I_{2}=\frac{2 \alpha_{0} \omega}{\alpha_{1} \sqrt{\alpha_{1}^{2}+4 \omega^{2}}}, \theta=\tan ^{-1} \frac{\alpha_{1}}{2 \omega}$ and $\alpha_{o}=\frac{d}{2 L}$

$I_{3}$ and $\phi$ are determined by the conditions that at $t=0, i=0$ and $\int_{i_{2}} d t=0$. These give:

$$
I_{3}=\frac{2 \alpha_{0} \sqrt{\alpha_{1}^{2} \omega^{2}+\left(\alpha_{1}^{2}+2 \omega^{2}\right)^{2}}}{\alpha_{1}\left(\alpha_{1}^{2}+4 \omega^{2}\right)}
$$

and

$$
\phi=\tan ^{-i} \frac{\alpha_{1} \omega}{\alpha_{1}^{2}+2 \omega^{2}}
$$

The complete expression for the current is therefore:

$$
\begin{gathered}
i=Q_{o} \frac{\omega_{o}^{2}}{\omega} \varepsilon^{-\alpha_{1} t} \sin \omega t-\frac{2 \alpha_{o} \omega}{\alpha_{1} \sqrt{\alpha_{1}^{2}+4 \omega^{2}}}\left\{\sin \left(\omega t+\tan ^{-1} \frac{\alpha_{1}}{2(\omega)}\right)\right. \\
\left.-\frac{\sqrt{\alpha_{1}^{2} \omega^{2}+\left(\alpha_{1}^{2}+2 \omega^{2}\right)^{2}}}{\omega \sqrt{\alpha_{1}^{2}+4 \omega^{2}}} \varepsilon^{-\alpha_{1} t} \sin \left(\omega t+\tan ^{-1} \frac{\alpha_{1} \omega}{\alpha_{1}^{2}+2 \omega^{2}}\right)\right\} \ldots
\end{gathered}
$$

This expression might not, at first inspection, seem to support fully my earlier statement that the expressions for the oscillatory current voltage and charge are simple and convenient. When, however, this expression is examined in the light of the fact that the ratio $\frac{\omega_{o}}{\omega}$ is almost exactly unity for slightly or moderately damped oscillators, and is very nearly unity even in the case of very heavily damped oscillators and when further account is taken of the fact that the ratio $\frac{\alpha_{1}}{2 \omega}$ is a very small quantity, even in the case of heavily damped oscillators, it is seen that the expression for the oscillatory current reduces to:

$$
i=\left\{Q_{o} \omega_{o} \varepsilon^{-a_{1} !}-\frac{\alpha_{o}}{\alpha_{1}}\left(1-\varepsilon^{-a_{1} t}\right)\right\} \sin \omega_{o} t
$$

In the limiting case of $\alpha_{1}=0$, or no damping except that due to the spark this expression reduces to

$$
i=\left(Q_{o} \omega_{0}-\alpha_{o} t\right) \sin \omega_{0} t
$$

which is the case of linear decrement treated in my first paper on the effect of the spark on the oscillations of an electric oscillator.

In the other limiting case, namely that in which $\alpha_{o}$ is negligible compared to $\varkappa_{1}$, the expression for the oscillatory current reduces to:

$$
i=Q_{o} \frac{\omega_{0}^{2}}{\omega} \varepsilon^{-a_{1} t} \sin \omega t
$$


which we immediately recognize as the well-known case of logarithmic decrement, first treated by Lord Kelvin and in which there is no damping due to the spark.

Before proceeding further with the specific subject of this paper, I shall call attention to the peculiar relations between $\omega_{0}$, $\omega$ and $\alpha_{1}$ which resulted in the marked simplification of the expression (9) for the oscillatory current.

If we define the logarithmic decrement per cycle of the oscillator in the absence of spark resistance as $\delta=\frac{\pi R}{L \omega}$, then the fact that $\frac{\omega_{0}}{\omega}$ is practically unity for all values of $\delta$ is exhibited in Table I.

TABLE I

\begin{tabular}{r|l|l|l|l|l|l|l}
\hline \hline$\delta=$ & 0.2 & 0.25 & 0.3 & 0.35 & 0.4 & 0.45 & 0.5 \\
$\frac{\omega_{o}}{\omega}=$ & 1.00050 & 1.00078 & 1.00114 & 1.00155 & 1.00202 & 1.00257 & 1.00317 \\
\hline
\end{tabular}

The fact that $\frac{\alpha}{2 \omega}$ and $\frac{\alpha \omega}{\alpha^{2}+2 \omega^{2}}$ are both small compared to unity and practically equal to each other over a wide range of values of $\delta$ including even cases of high damping is exhibited in Table II.

TABLE II

\begin{tabular}{r|r|r|r|r|r|r|r|r|r|r|r|r|}
\hline$\delta=$ & 0.1 & 0.15 & 0.2 & 0.25 & 0.3 & 0.35 & 0.4 & 0.45 & \multicolumn{1}{l}{0.5} \\
$\tan -1 \frac{a}{2 \omega}$ & .0080 & .0120 & .0160 & 0.020 & 0.0240 & 0.0280 & 0.0320 & 0.0360 & 0.040 \\
& $0^{\circ} 27^{\prime}$ & $0^{\circ} 41^{\prime}$ & $0^{\circ} 54^{\prime}$ & $1^{\circ} 9^{\prime}$ & $1^{\circ} 22^{\prime}$ & $1^{\circ} 35^{\prime}$ & $1^{\circ} 49^{\prime}$ & $2^{\circ} 3^{\prime}$ & $2^{\circ} 17^{\prime}$ \\
$\tan -1 \frac{a \omega}{a 2+2 \omega 2}$ & 0.0080 & 0.0120 & 0.0160 & 0.020 & 0.0240 & 0.0280 & 0.0320 & 0.0360 & 0.040 \\
\hline
\end{tabular}

This digression is made and these tables are here introduced because the relations involved are of such universal application in the theory of oscillators and are capable of producing such marked simplifications of the mathematical expressions and computations therefrom, that it is well to have on record for convenient reference the numerical relations of these functions over a fairly wide range of values of the logarithmic decrement $\delta$.

The expression for the quantity of electricity involved in the oscillations, when this expression is simplified in the same way as that for the current, becomes:

$$
q=\left\{\left(Q_{o}+\frac{\alpha_{o}}{\alpha_{1} \omega_{o}}\right) \varepsilon^{-a_{1} t}-\frac{\alpha_{o}}{\alpha_{1} \omega_{o}}\right\} \cos \omega_{o} t
$$


The voltage is, of course, merely $v=\frac{q}{C^{\prime}}$, where $C$ is the capacity of the condenser.

The expression for the current enables us to determine one for the resistance $R_{2}$ of the spark in terms of its initial resistance $R_{0}$ and time for, save in the very exceptional cases to which this theory does not apply, the resistance of the spark is inversely proportional to the amplitude of the current.

If we designate this amplitude by $I$, we have:

and

$$
\begin{gathered}
I=Q_{o} \omega_{o} \varepsilon^{-a_{1} t}-\frac{\alpha_{o}}{\alpha_{1}}\left(1-\varepsilon^{-a_{1} t}\right) \\
R_{2}=R_{o} \frac{I_{o}}{I}
\end{gathered}
$$

where $I_{o}$ is the initial amplitude of the current or $Q_{0} \omega_{0}$.

These expressions shed considerable light on the quenching of the oscillations of a wave train after a certain time $T$. In the logarithmic decrement theory there was no indication of the time of quenching, the current merely indefinitely diminished, and tho experience taught us that after a relatively definite time for any particular oscillator, the oscillations positively ceased and the circuit automatically opened at the spark gap, yet there was no recognition of this fact in that theory.

An important result of the fact that this theory which we have just developed, takes cognizance of the definite finite length of an oscillation train, is that it enables us to express in a Fourier's series any isochronous succession of similar damped oscillation trains, provided the oscillation trains do not overlap. The expression in a sine series is effected in this case, as in the case of the linear decrement theory. ${ }^{3}$

In determining the co-efficients of the series it is sufficient to take into consideration only the amplitude of the successive oscillation trains and not the instantaneous value of the oscillations, so that the series is of the form:

$$
i=\sin \omega_{o} t \sum_{1}^{\infty} a_{m} \sin \frac{m \pi}{T_{1}} t
$$

where $T_{1}$ is the periodic time of recurrence of the oscillation trains or the reciprocal of the group or spark frequency.

An examination of the expression (9) for the current shows that after a time

${ }^{3}$ Proceedings of The Institute of Radio Engineers, Volume 2, Number 4, page 318. 


$$
T_{o}=\frac{1}{\alpha_{1}} \log _{\varepsilon}\left(Q_{o} \frac{\alpha_{1} \omega_{o}}{\alpha_{o}}+1\right)
$$

the amplitude of the current becomes zero and therefore by (15) the resistance of the spark gap becomes infinite. This time $T_{o}$, is obviously the time of the quenching of the oscillations.

If $T$ is the time of a complete oscillation, then $\frac{T_{o}}{T}$ is evidently the number $N$ of complete oscillations in an oscillation train, so that

$$
N=\frac{\omega_{o} \log _{\varepsilon}\left(Q_{o} \frac{\alpha_{1} \omega_{o}}{a_{o}}+1\right)}{2 \pi a_{1}}
$$

Concerning the condition of non-oscillatory discharge, evidently this occurs when $T_{o}=\frac{T}{2}$ so that the condition is reached when

$$
\frac{\alpha_{1}}{a_{o}}=\frac{\frac{\pi a_{1}}{\omega_{o}}-1}{I_{o}}
$$

The first of the last two expressions shows that $\alpha_{o}, \alpha_{1}$, and $\omega_{o}$ remaining the same, the persistency of oscillations increases with the quantity of electricity discharged across the gap.

This expression (18) also shows that $Q_{o}$ and $\frac{\omega_{o}}{a_{1}}$ remaining the same, the persistency of the oscillations decreases with the ratio $\frac{\alpha_{o}}{\alpha_{1}}$.

Similarly, the expression (19) shows that a circuit which is just aperiodic may become oscillatory thru an increase in the quantity of electricity discharged across the gap. This increase of $Q_{o}$ of course means increase in the ratio $\frac{C}{L}$.

Table III shows the variation in the number of complete oscillations per oscillation train for varying values of $\frac{\alpha_{o}}{\alpha_{1}}$.

In this table the remaining quantities are taken as

$$
Q_{o}=10^{-15},(1)=()_{o}=10^{6} \text { and } \delta=0.2 \text {. }
$$

\section{TABLE III}

\begin{tabular}{l|c|c|c|c|c|c|c|c|c|c}
\hline$\underline{a}_{0}=$ & 0.1 & 0.5 & 1 & 2 & 3 & 4 & 5 & 6 & 7 & 8 \\
$a_{1}$ & 23.1 & 15.0 & 12.0 & 8.96 & 7.33 & 6.27 & 5.49 & 4.91 & 4.44 & 4.05 \\
\hline
\end{tabular}

Figure 1 shows the decay of the amplitude of the oscillations 
of this same oscillator for various values of the ratio $\frac{\alpha_{0}}{a_{1}}$. The outermost curve of this figure shows the decrescence of amplitude for the case of $\frac{\alpha_{0}}{a_{1}}=0.1$, the next curve corresponds to the ratio $\frac{\alpha_{o}}{\alpha_{1}}=0.5$, etc., to the innermost curve which corresponds to a ratio $\frac{\alpha_{0}}{\alpha_{1}}=9.0$.

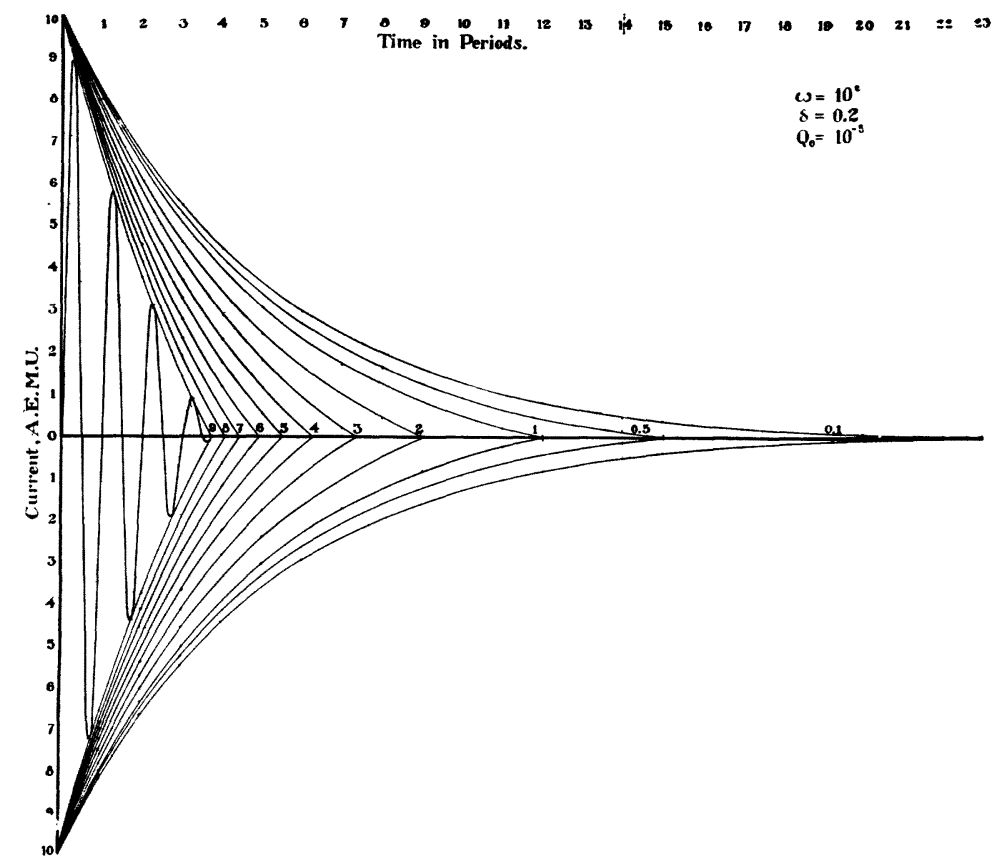

Figure 1

Table 4 shows the variations in the number of complete oscillations per oscillation train for varying values of the quantity $Q_{o}$ of electricity involved in the oscillations. In this table the other constants of the oscillator involved are $\omega=\omega_{0}=10^{6}$, $\grave{o}=0.2$ and $\frac{\alpha_{o}}{\alpha_{1}}=1$

TABLE IV
\begin{tabular}{c|c|c|c|c|c}
$Q_{o}=$ & 0.0001 & 0.0002 & 0.0003 & 0.0004 & 0.0005 \\
$N=$ & 12.0 & 15.2 & 17.2 & 18.6 & 19.7 \\
\hline
\end{tabular}


Figure 2 shows the decrescence of the amplitude of the oscillations of an oscillator having these constants, for varying values of $Q_{0}$.

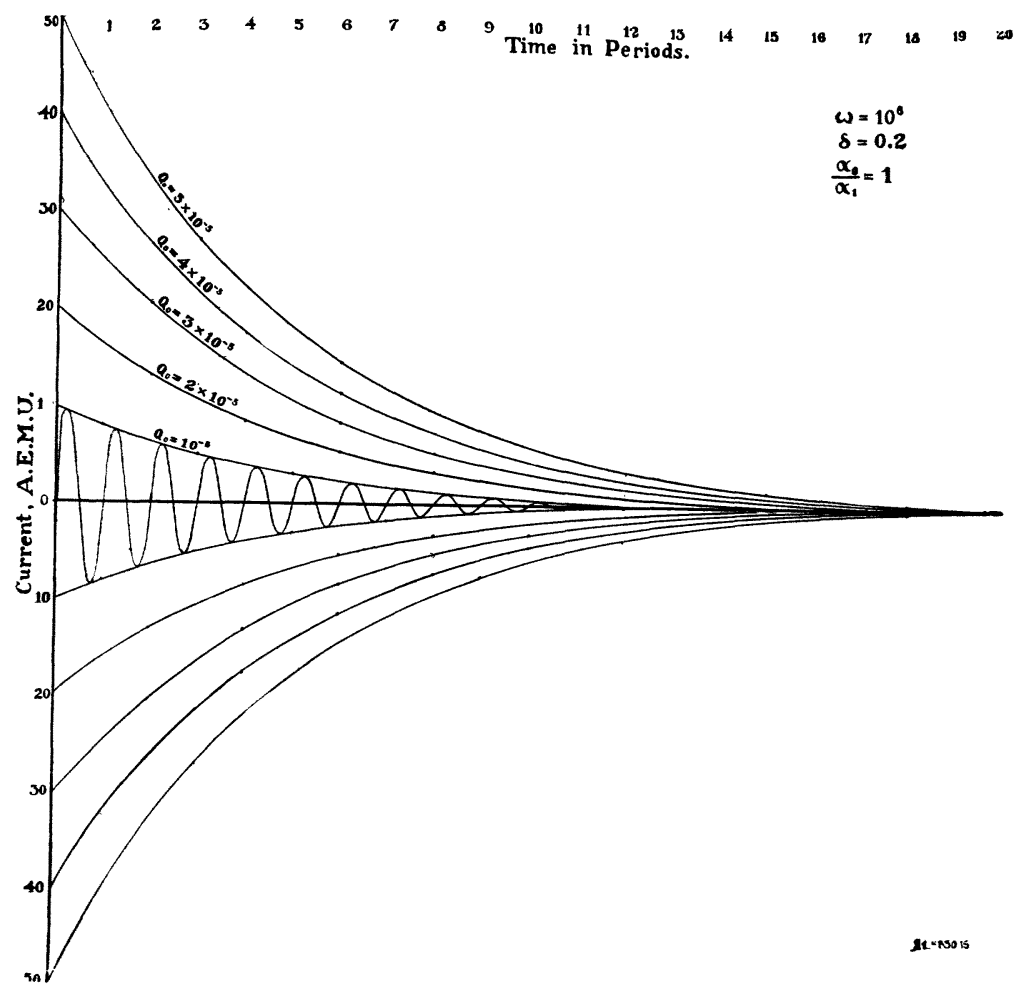

FIGURe 2

The increase of resistance of the spark with time for different values of $\frac{\alpha_{o}}{\alpha_{1}}$ and for different values of $Q_{o}$ is exhibited in Figure 3. The curves marked $0.1,0.5,1,2$, etc., to 8 , each represent the spark resistance for the corresponding ratio of $\frac{\alpha_{o}}{\alpha_{1}}$, the remaining constants of the oscillator being $Q_{o}=10^{-5}, \omega=\omega_{o}=10^{6}$ and $\delta=0.2$. The curves marked $Q_{o}=10^{-5}, Q_{o}=2 \times 10^{-5}, Q_{o}=3 \times 10^{-5}$, etc., represent the spark resistance for corresponding values of $Q_{o}$, the other constants being $\omega=\omega_{o}=10^{6}, \frac{\alpha_{o}}{a_{1}}=1$ and $\delta=0.2$.

In my earlier paper on the subject of the resistance of the spark, the mode of variation of the resistance of an arc with the 
instantaneous value of the current flowing thru it and the hysteretic lag in the resistance of an alternating current are is

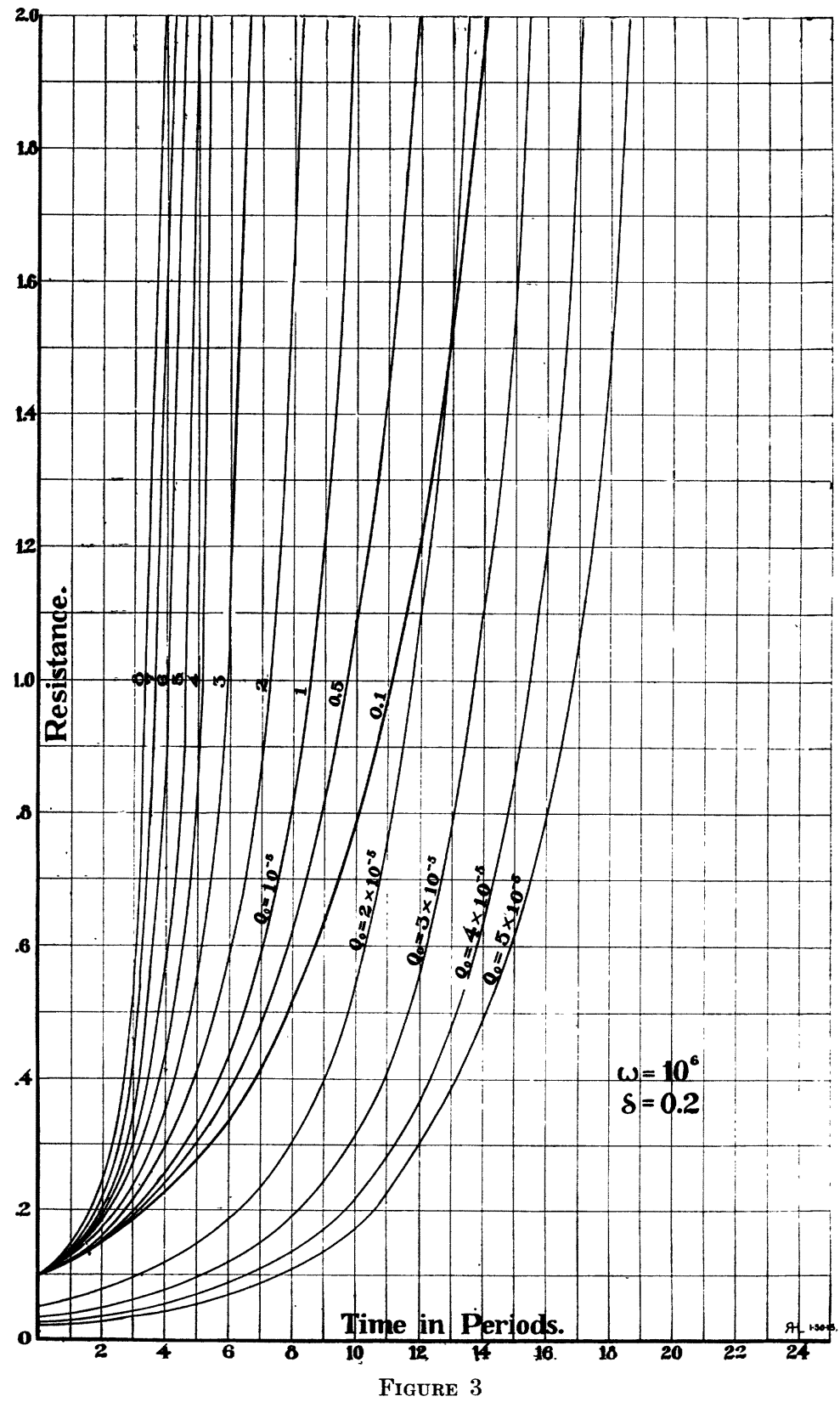


discussed. ${ }^{4}$ The effect of these factors upon the shape of the curve of the instantaneous values of the voltage at the terminals of the are is illustrated in Figure 8 of that paper. It is there shown that as the frequency of the alternating current is progressively increased, the instantaneous value of the resistance of the arc or spark becomes more and more nearly independent of the instantaneous value of the current and progressively tends to become more nearly inversely proportional to the amplitude of the alternating current so that when radio frequencies are reached, the simple law of inversed ratio to the amplitude of the current, in general, obtains.

In order to determine, in any particular case, to what extent, if any, there is a deviation in the resistance of the spark from the law of simple inverse ratio to amplitude of current, it is sufficient to examine the oscillators, by means of an extremely loosely coupled exploring resonant circuit, for the first odd harmonic. The relative magnitude of this odd harmonic, if such be found, to the fundamental oscillation will be an index of the departure, if any exists, from the law of simple inverse proportionality of the spark or arc resistance to the amplitude of the current.

Figure 4 of this paper shows how the peculiar dissymmetrical curve of instantaneous voltage across an alternating current arc may be synthetically constructed by the addition to the fundamental sine curve, of the two first odd harmonics. In this figure, curve 3 is the sine curve of the fundamental. Curve 4 is the sine curve of the first odd harmonic, curve 5 is the sine curve of the second odd harmonic, curve 6 is the cosine curve of the fundamental, curve 7 is the cosine curve of the first odd harmonic, the symmetrical curve 1 is the sum of curves 3,4 , and 5 , and the dissymmetrical curve 2 is the sum of $3,4,5,6$, and 7 .

Curve 1 corresponds very closely in shape to that of the voltage across an alternating current are in which the instantaneous values of the resistance varies according to the wellknown law

$$
\rho=\frac{a}{i}+\frac{b}{i^{2}}
$$

which obtains for unidirectional ares and for ares in which the current varies so slowly as not to exhibit the effects of the hysteretic lag of resistance behind current variation.

In this expression, which is the same as that given on page 320 of my previous paper, $\rho$ is the instantaneous value of the

${ }^{4}$ Proceedings of The Institute of Radio Engineers, Volume 2, Number 4 , pages 320 to 322 . 
resistance of the arc or spark in question, $a$ and $b$ are constants and $i$ is the current. This expression for the resistance of an arc or spark may be said to give its "static resistance."

Curve 2, on the other hand, corresponds very closely to the curve of the instantaneous voltage across an alternating current arc of ordinary lighting frequencies in which the effect of the resistance hysteresis is well defined.

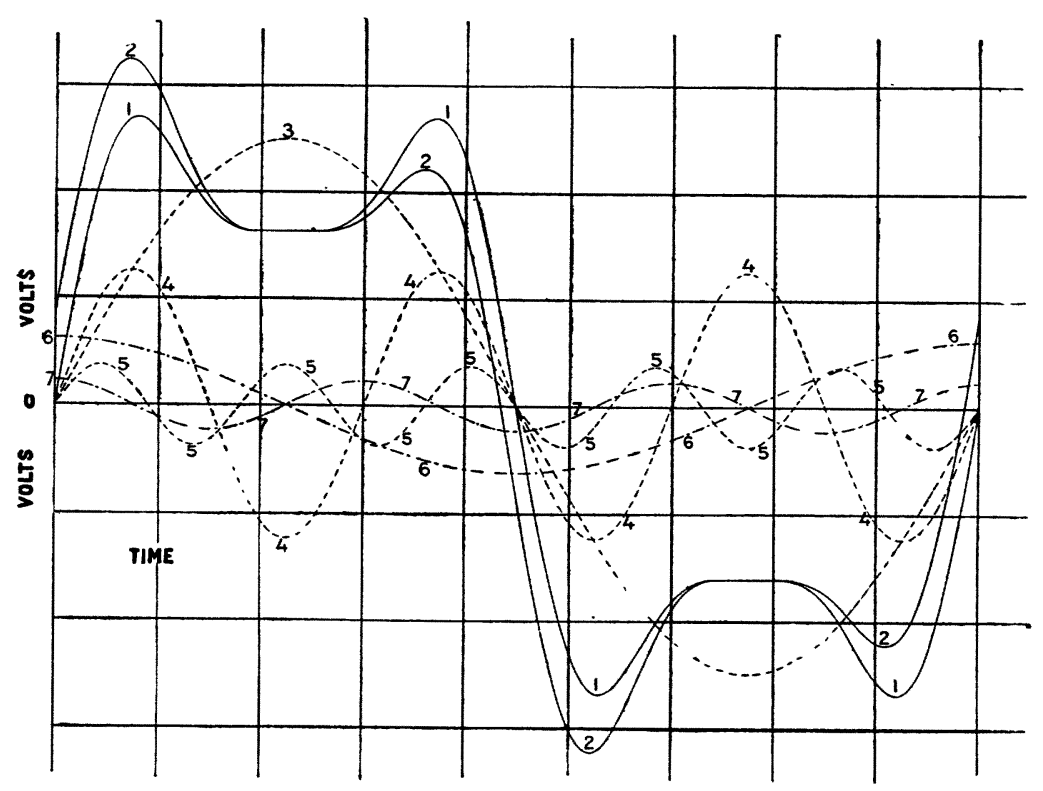

Figure 4

It will be noted that the two symmetrical peaks of curve 1 , with the flat valley between them, are produced by the addition to the sine curve of the fundamental, of the sine curves of the first and second odd harmonics, while the dissymmetry of the peaks of curve 2 and the slight shift in phase of that curve is due, almost wholly, to the addition of the cosine curve of the fundamental. The diagram of Figure 4 illustrates well, therefore, how a resonant harmonic analysis of the oscillations of a circuit may be used to shed light on the degree to which, if at all, the resistance of the spark depends upon the instantaneous values of the current and therefore differs from the law of simple inverse proportionality to the amplitude of the current. It 
shows, moreover, that the degree to which the hysteresis of the spark or arc resistance is effective is not so easily determined.

Coming now to one of the practical applications of some of the considerations contained in my two papers on the resistance of the spark and its effect on the oscillations of electric circuits, it is to be noted that the quenching of a spark or arc is the same thing as the development of an infinite or practically infinite gap resistance. In the case of an alternating arc or spark, there are only two reasons why the resistance of the gap does not reach an infinite value every time the current passes thru a zero value, i.e., once for each alternation or reversal. The first reason is the resistance hysteresis and the second is that the current does not remain long enough at or near its zero value to overcome the effect of the resistance hysteresis and permit the gap to assume its "static resistance" in accordance with the expression (20).

The hysteretic lag of the resistance of an arc or spark behind the instantaneous value of current change may be minimized by anything that tends to cool the arc or spark gap quickly, or otherwise to extinguish the arc or spark. For this purpose air blasts and powerful magnetic fields across the gap have been successfully used. The characteristic structure now so wellknown as the quenching gap, giving as it does the maximum mechanical opportunity for the conduction and radiation of the heat away from the spark as well as the sub-division of the spark into a number of small series sparks, is much used. The use of electrodes of sueh metals as silver and copper, which minimized the hysteretic lag of the resistance of the spark, and the use of hydrogenous vapor at the gap are common. But besides minimizing the hysteresis of the spark, it is customary in the quenched spark systems to cause two oscillations of approximately equal amplitude and differing from each other in frequency by a small percentage, to pass across the gap at each spark so that owing to the beats between these oscillations, the amplitude of the oscillating current across the gap will fall to zero after say about five oscillations or so have passed and the resistance of the gap thereby permitted to attain a practically infinite value in spite of such resistance hysteresis as the spark may have.

Up to the present time most effort has been exerted to the diminution of the resistance hysteresis of the spark, i. e., to the enhancing of the quenching action at the gap per se. But it is evident from the aspect of the subject as here presented that when great or rapid quenching is required as in the production of impulsive excitation or in the production of sustained oscillations, 
it is quite as important to provide time for the hysteresis of the arc or spark to expend itself and allow the gap to attain its "static resistance" as it is to minimize the resistance hysteresis at the gap. This can only be done by providing intervals of time when the current across the gap is zero or practically zero, these intervals being made long enough to permit the resistance at the gap to become sensibly infinite.

To accomplish this result we may have recourse to a combination of alternating currents or oscillations simultaneously flowing across the gap, these alternating currents or oscillations being so chosen that they will combine, together and with the supply current, to form intervals of zero current or substantially zero current across the gap.

By Fourier's theorem, it is evident that we can produce a combination of alternating currents, which will satisfy this requirement with as great a degree of approximation as we may wish. Thus the series:

$$
i=I_{o}\left\{1+\frac{4}{\pi}\left(\sin \frac{2 \pi}{T} t+\frac{1}{3} \sin \frac{6 \pi}{T} t+\frac{1}{5} \sin \frac{10 \pi}{T} t+. .\right)\right\}
$$

is a function which alternately has the values $2 I_{o}$ and 0 , holding each of these values for successive intervals of time $\frac{T}{2}$. The degree of approximation to the desired result which may be obtained by this series when using four of the sine terms, is illustrated in Figure 5. In this diagram curve 1 is the sum of all the dotted sine curves, $2,3,4$, and 5 , taken about axial line $I_{o}$. These dotted curves may be taken to represent alternating or oscillating currents and the straight line $I_{o}$ may be taken to represent a steady unidirectional charging current. It will be noted that for the first half of the period the resultant current represented by curve 1 is approximately $2 I_{o}$ in value, and for the second half it is approximately zero. Such a combination of currents passing across a spark gap would cause it to quench once in each half period even were the fundamental alternating or oscillating current of radio frequency.

If it is desired to make the interval of time during which the current is approximately zero long compared to that during which it is at full strength, another series of alternating currents may be deduced by Fourier's theorem, but for practical purposes it would not be desirable or necessary to have resort to a Fourier's series, which would lead to the necessity of using too great a number of different alternating or oscillating component currents. One who 
is reasonably familiar with the practical working of Fourier's series can work out much simpler combinations of alternating or oscillating currents, which will give the desired intervals of approximately zero current with a sufficient degree of precision for

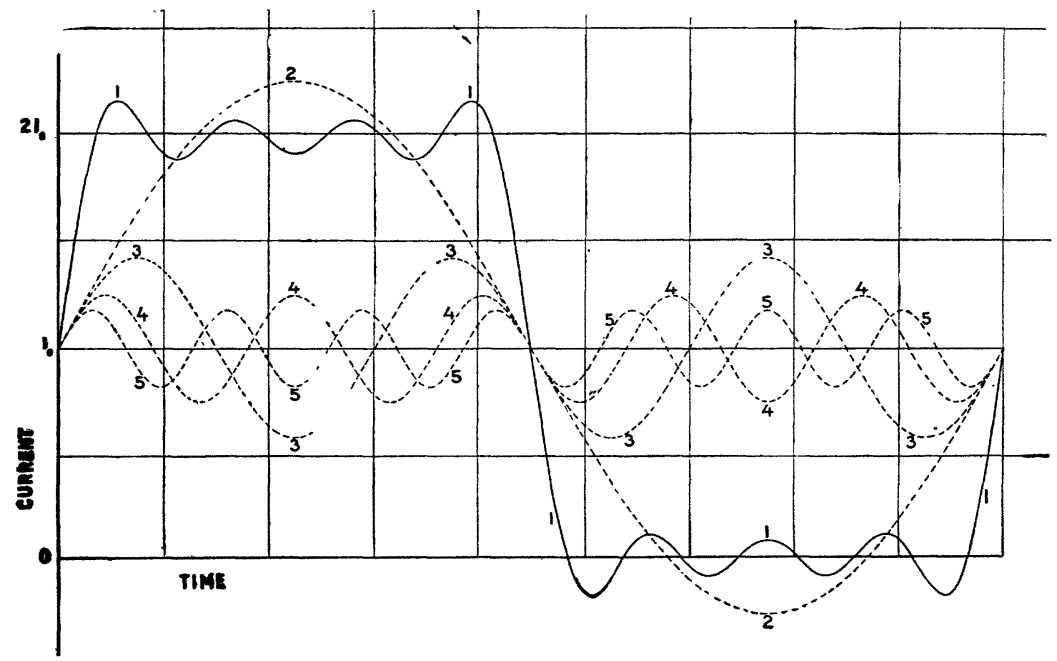

Figure 5

practical purposes. As an example, Figure 6 shows a case where the gap current illustrated by curve 3 is approximately zero $5 / 9 t h s$ of the half period. This result is accomplished by the

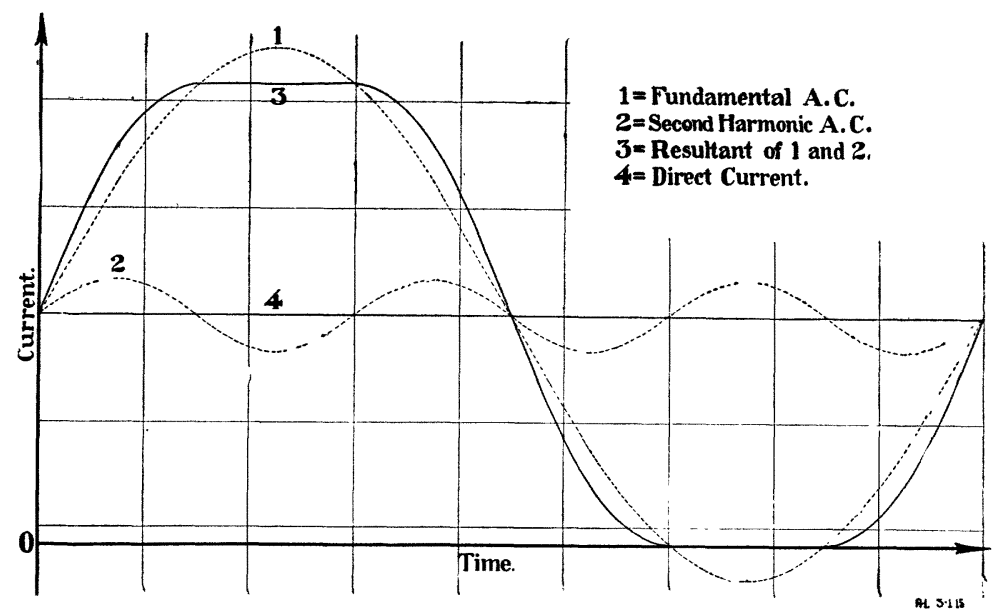

Figure 6 
combination of a unidirectional charging current and two alternating or oscillating currents, the equation for the gap current being:

$$
i=0.86+\sin \omega t+0.135 \sin 3 \omega t
$$

Perhaps a better arrangement might be secured for some purposes by the use of three frequencies, the equation for the gap current then being approximately

$$
i=0.80+\sin \omega t+0.26 \sin 3 \omega t+0.064 \sin 5 \omega t
$$

This case is illustrated in Figure 7 and it is seen that the gap current is approximately zero for two-thirds of the half period. Probably the easiest way to secure impulsive excitation, how-

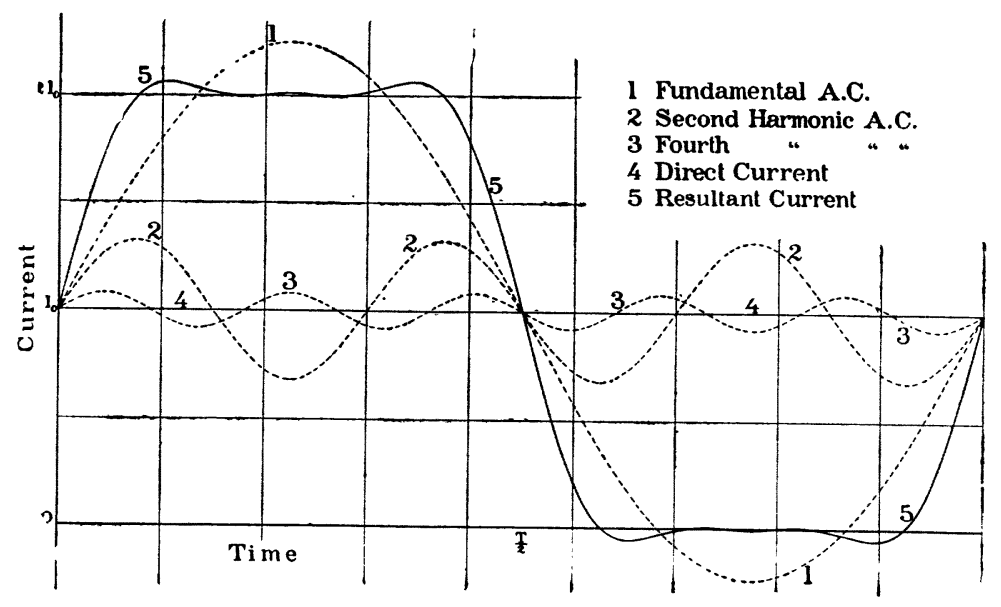

Figure 7

ever, would be to employ a supply current not great enough, per se, to maintain an arc across the gap and then use a combination of oscillating or alternating currents whose sum would be zero over a considerable fraction of the time period of the fundamental. Such a combination is given approximately by the series:

$$
\sin \omega t+\sin 2 \omega t+\frac{1}{3} \sin 3 \omega t
$$

This series is approximately zero for about $4 / 11$ ths of the total period.

Several ways in which these combinations of gap currents may possibly be secured in the case where oscillating circuits are 
used and some suggestive modes of associating the oscillating circuits with the aerial are shown in Figures 8, 9, 10, 11 and 12, where $S$ represents a quench gap which may have its quenching power enhanced, if necessary, by the use of hydrogenous vapor between its plates and by the use of a powerful magnetic field acting axially along the gap.

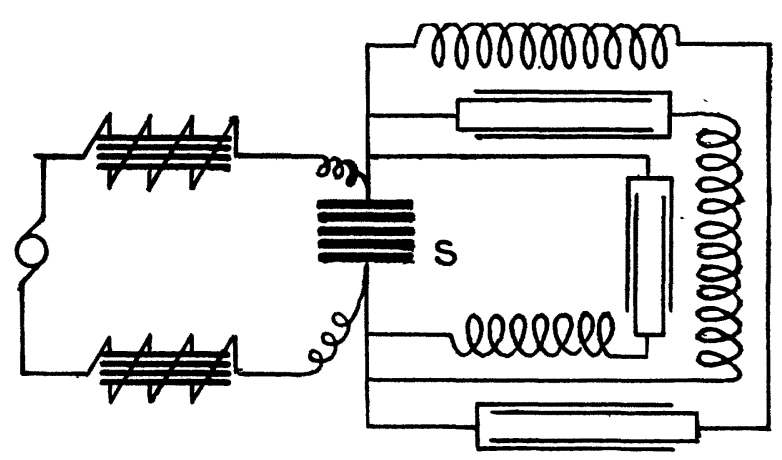

Figure 8

No attempt can be made here to go into a detailed discussion of the theory of operation of such circuits. Suffice it to point out that when there is more than one oscillating circuit shunted about a gap, the quenching of the spark after the initial surge has passed leaves the remaining circuits free to oscillate without the damping effect of the spark. If there be two oscillation circuits, these residual oscillations will have but a single frequency. If there be three oscillation circuits these oscillations will have three frequencies. If there are four there will be six frequencies, etc., the number of frequencies being given by the expression

$$
N_{1} \frac{N_{1}-1}{2}
$$

where $N_{1}$ is the number of branch oscillation circuits. Of course, if the aerial or radiating circuits be one of the oscillating circuits shunted about the gap, it tends to develop additional higher frequency component currents which are not, strictly speaking, harmonics, tho always of higher frequency than the main or fundamental natural frequency of the aerial. This phenomena may, in some instances, be of sufficient importance to modify, somewhat, the proportion of the other circuit or circuits shunted about the gap for the purpose of producing impulsive excitation of the aerial. These high frequencies can be suppressed to a 
very considerable degree by working the aerial at a frequency much below its fundamental natural frequency. Moreover, when the aerial is one of the oscillating circuits shunted about

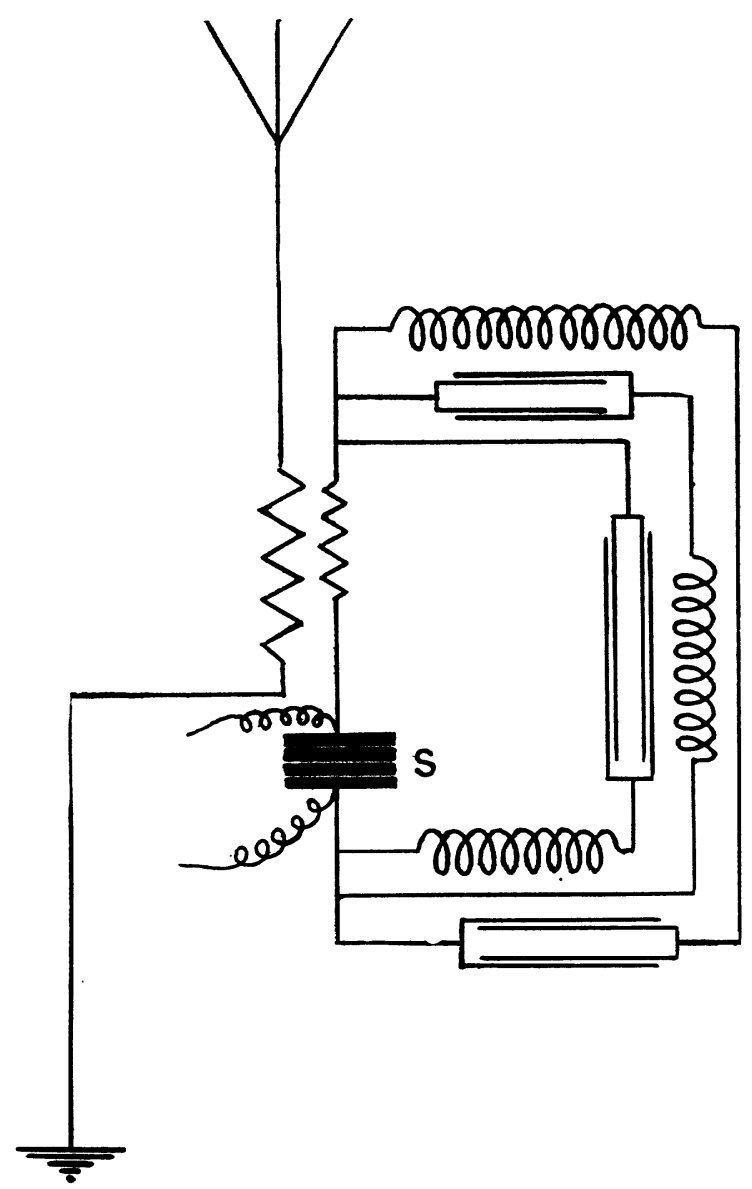

Figure 9

the gap, and a single wave length of radiation is desired, it will, in general, be necessary to employ but a single additional circuit shunted across the gap.

When two spark gaps are used in parallel as shown in Figure 12 , the sparks occur alternately in the two gaps and if the gaps be shunted by similar oscillators, the oscillations developed in them will be opposite in phase. By this means, two trains of oscillations having any desired phase relation to each other 


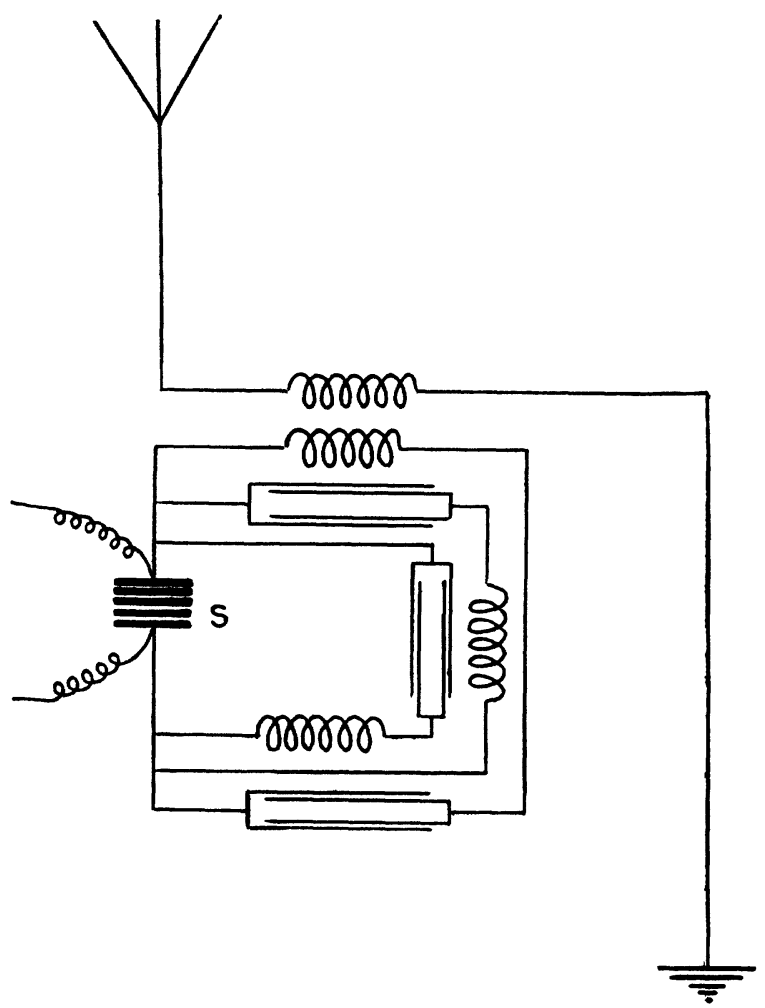

Figure 10

should be attainable. Furthermore, in this arrangement of parallel spark gaps, it should be possible to maintain the supply current extremely constant and to secure for a given power much higher frequencies of oscillation than with a single gap.

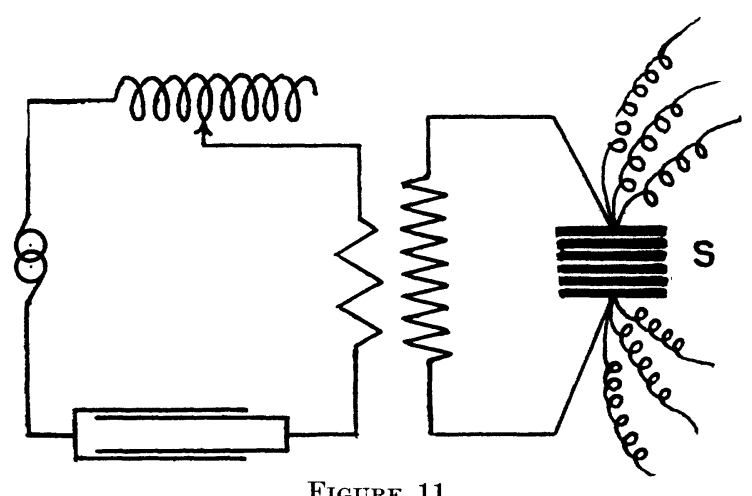

Figure 11 


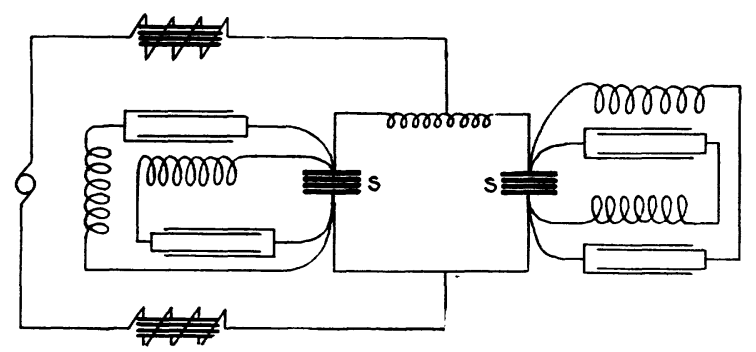

FigURE 12

SUMMARY: The Thompson or "logarithmic decrement" theory of oscillatory circuits is contrasted with the linear decrement theory. Both are limiting cases of the more general theory which considers circuits containing both conductor and spark resistance. It is this last and most general theory which is fully developed in the present paper.

It is found that the effect of the spark is to introduce a simple harmonic counter E. M. F. of constant amplitude during the oscillations and having the same frequency as these oscillations. The mathematical expressions for the current and voltage, under practical working conditions, are found to be simple, and to reduce to the older expressions in the limiting cases.

The new theory gives the value of the spark resistance at any time and determines the quenching time. A new resonance analysis method of studying the law governing the dependence of spark or arc resistance on current is given.

A novel method of producing "quenching" in gaps is the following. There are shunted around the gaps several circuits of different frequencies so proportioned that the total current remains appreciably zero for a considerable portion of each cycle (of the fundamental frequency). The practical applications of this method to radio telegraphy are described in detail. Finally, a parallel spark gap method for obtaining oscillations of any desired phase difference is given. 